\title{
Visual onset expands subjective time
}

\author{
RYOTA KANAI \\ University of Utrecht, Utrecht, The Netherlands \\ and California Institute of Technology, Pasadena, California \\ and \\ MASATAKA WATANABE \\ University of Tokyo, Tokyo, Japan
}

\begin{abstract}
We report a distortion of subjective time perception in which the duration of a first interval is perceived to be longer than the succeeding interval of the same duration. The amount of time expansion depends on the onset type defining the first interval. When a stimulus appears abruptly, its duration is perceived to be longer than when it appears following a stationary array. The difference in the processing time for the stimulus onset and motion onset, measured as reaction times, agrees with the difference in time expansion. Our results suggest that initial transient responses for a visual onset serve as a temporal marker for time estimation, and a systematic change in the processing time for onsets affects perceived time.
\end{abstract}

Our estimation of time does not always correspond to physical reality, but is subject to distortion, depending on the temporal configuration of the stimulus (Arao, Suetomi, \& Nakajima, 2000; Nakajima, ten Hoopen, Hilkhuysen, \& Sasaki, 1992; Nakajima et al., 2004; Nakajima, ten Hoopen, \& van der Wilk, 1991; Rose \& Summers, 1995) or the observer's internal state, such as the state of attention (Brown, 1985, 1997; Coull, Vidal, Nazarian, \& Macar, 2004; DeWolfe \& Duncan, 1959; Tse, Intriligator, Rivest, \& Cavanagh, 2004; Zakay, 1993). Moreover, our voluntary action is also known to distort perceived time (Yarrow, Haggard, Heal, Brown, \& Rothwell, 2001; but see Alexander, Thilo, Cowey, \& Walsh, 2005).

One of the most influential models of time perception is the scalar expectancy theory, or SET (Gibbon, 1977; Gibbon, Church, \& Meck, 1984). The SET model consists of several functional components. The first component is a clock process - a pacemaker generating pulses (Creelman, 1962; Treisman, 1963). The pulses are gated by a switch, which is controlled by time markers indicating the beginning and the end of an interval to be estimated. While the switch is open, the pulses accumulate in working memory, representing the online estimation of the duration. After the switch closes, the accumulated pulses, which correspond to the total duration estimated, are transferred to a more long-lasting reference memory, available for later comparison. The comparison process evaluates the duration previously stored in reference memory and the online estimate of a duration accumulated in working memory.

We thank Daw-An Wu, Concetta Morrone, Shin'ya Nishida, David Rose, and Shinsuke Shimojo for discussion and comments on an earlier version of the manuscript. Correspondence concerning this article should be sent to R. Kanai, California Institute of Technology, M/C 139-74, Pasadena, CA 91125 (e-mail: kanair@caltech.edu).
The result of this comparison is used for making relative time judgments.

This model suggests that there are several factors that can lead to a distortion of time perception. First, the opening and closing of the switch gating the pulses might involve some processing delays. When the duration of an event is estimated, the beginning and end of the event need to be marked. If the opening of the switch were delayed, the estimated duration would become shorter. Similarly, if the closing of the switch were delayed in comparison with the opening, the estimated duration would be lengthened further. Systematic manipulations of the delays would result in a time distortion (see, e.g., Yarrow, Haggard, \& Rothwell, 2004). Second, the rate of the pulses is not necessarily constant. Although SET does not specify what constitutes the pulse generation, both attention and the number of changes experienced by observers seem to play an important role here. For example, it has been suggested that attention and arousal increase the speed of the internal clock. This account has been applied to the past findings that when a person directs his or her attention to temporal events, the perceived duration becomes longer (Brown, 1985, 1997; Coull et al., 2004; DeWolfe \& Duncan, 1959). It has been proposed that changes in a stimulus lengthen perceived duration (Block, 1982; Gibson, 1975; Poynter, 1989). For example, intervals containing many events or movements (i.e., changes in position) are perceived to be longer than those involving fewer events (Brown, 1995; Kanai, Paffen, Hogendoorn, \& Verstraten, 2006).

The focus of our present study is time distortion associated with sensory components. We investigated how different types of visual time markers influence our time perception. Past studies have shown that the characteristics of the stimuli marking an interval can affect time perception. In the auditory domain, interval duration discrimination is better when the onset and offset of the interval are marked 
by short stimuli $(<150 \mathrm{msec}$ ) than when they are marked by long stimuli ( $>225 \mathrm{msec}$; Rammsayer \& Leutner, 1996). Also, time perception depends on the modality marking the interval. When the onset and the offset of an interval are defined by different modalities (e.g., audition and vision), discrimination performance deteriorates in comparison with the case in which the markers are within the same modality (Grondin, Ivry, Franz, Perreault, \& Metthé, 1996; Grondin \& Rousseau, 1991). Finally, when the duration of the time marker becomes longer, the perceived duration of the interval increases (Grondin et al., 1996). These studies suggest that the gating mechanisms mediated by the switch play a role in the distortion of time perception.

In the present study, we examined whether the time marker defined by the appearance of a new visual object (hereafter referred to as "stimulus onset") differs from the time marker defined by a change in feature ("motion onset"). There is some evidence suggesting a difference in the processing for these two types of events. For example, a study on the attentional blink - a brief impairment in identifying and reporting a second target presented just following an earlier target stimulus - shows that the onset of a new stimulus is fundamentally different from a change in feature. Although the appearance of a new object produces an attentional blink, a change in a feature does not (Raymond, 2003). The difference in the attentional response between the two types of onset markers may result in a different delay for the switch process.

We compared the perceived duration of a time interval defined by the abrupt appearance of a stimulus versus the perceived duration of an interval whose onset was defined by a change in a stimulus feature (i.e., motion). Our typical stimulus consisted of two successive intervals (see Figure 1A), each defined by a particular direction of stimulus motion. In the first interval, the stimulus moves for a certain duration (480 $\mathrm{msec})$. In the second interval, which follows without any delay, the stimulus moves in the opposite direction for the same duration and then disappears. While keeping this stimulus configuration constant, we manipulated the visual time marker for the onset of the first interval. In one condition, the first interval started abruptly from a blank screen (i.e., the stimulus onset condition), and in the other condition, the first interval was preceded by a stationary stimulus that lasted between 1 and $2 \mathrm{sec}$ (the motion onset condition). Thus, the beginning of the first interval was defined by either stimulus appearance or motion onset, whereas the beginning of the second interval was always defined by motion reversal.

\section{GENERAL METHOD}

Stimuli were presented on a 22-in. monitor (LaCie Electron) controlled by a Macintosh G4 running MATLAB 5.2 (MathWorks, Inc.) and Psychophysics Toolbox (Brainard, 1997; Pelli, 1997). The refresh rate of the monitor was $75 \mathrm{~Hz}$ and the resolution was $1,024 \times$ 768 pixels. The viewing distance was $57 \mathrm{~cm}$. Head movements were limited by a chinrest.

The stimuli used in all experiments consisted of 200 coherently moving dots shown within an imaginary square aperture $\left(8^{\circ} \times 8^{\circ}\right)$, centered on the display. The speed was $6^{\circ} / \mathrm{sec}$. Each dot had a lifetime of $120 \mathrm{msec}$. In the first frame of a stimulus, each dot was randomly assigned a lifetime between $13 \mathrm{msec}$ (one frame) and $120 \mathrm{msec}$, to avoid the synchronous appearance and disappearance of the dots. After the lifetime of a dot had expired, a new moving dot appeared at a random position in the stimulus area. The luminance of the dots was $56 \mathrm{~cd} / \mathrm{m}^{2}$.

\section{EXPERIMENT 1 Basic Properties}

\section{Method}

Subjects. Four subjects participated. One of the subjects was an author (R.K.), but the other 3 observers were naive as to the purpose of the experiments. All of the observers had normal or corrected-tonormal visual acuity.

Procedure. The perceived duration of the first interval relative to the second interval was measured by the method of constant stimuli. We varied the duration of the second interval $(427,480,533,587$, 640 , and $693 \mathrm{msec}$ ) while keeping the duration of the first interval constant $(480 \mathrm{msec})$. The observers' task was to indicate which interval had the longer duration. The effect size was estimated by fitting a psychometric function to the data. The points where the fitted curves crossed $50 \%$ were taken as the points of subjective equality (PSEs), and the differences between the PSEs and the physical duration $(480 \mathrm{msec})$ were taken as the amount of time expansion of the first interval. Two conditions were examined: the stimulus onset condition and the motion onset condition (Figure 1A, top panels and bottom panels, respectively). These conditions were performed in separate blocks, with the order counterbalanced across observers.

In the stimulus onset condition, the observers initiated a trial by a keypress. After a randomized duration (uniform sampling between 1 and $2 \mathrm{sec}$ ), the stimulus appeared and immediately moved for $480 \mathrm{msec}$ in one direction (left or right), and then moved in the opposite direction for a duration that varied between $-53,0,53,107$, 160 , and $213 \mathrm{msec}$, with respect to the duration to the first interval. In the motion onset condition, the stimuli were identical except that a stationary stimulus appeared at the keypress, remained there for a random duration (uniform sampling between 1 and $2 \mathrm{sec}$ ), and then started moving. In both conditions, the task was to indicate which interval was longer in time. The direction of motion in the first interval was randomized between leftward or rightward. A psychometric function (a probit curve; see Kanai, Sheth, \& Shimojo, 2004) was fit on the data for each condition, and the point at which the curve intersects $50 \%$ was taken as the effect size.

Design. The subjects were tested individually for each condition of $2 \times 2$ within-subjects factorial design. The factors were onset type (stimulus onset or motion onset) and motion direction of the first interval (left or right). The two onset conditions were tested in separate blocks, and the left trials and right trials were intermixed within a block. For each of the four conditions, the amount of time expansion of the first interval relative to the second interval was estimated. Each subject performed the trials of the stimulus onset condition and motion onset condition in a separate block, and the order of the two blocks was counterbalanced across subjects.

\section{Results and Discussion}

The differences of the means of the PSEs were tested with a repeated measures ANOVA. No significant difference was found between the trials starting with leftward motion and the trials starting with rightward motion $[F(1,3)=2.435, p=.22]$. Thus, the data were averaged for the leftward and rightward trials for each observer.

Figure $1 \mathrm{~B}$ shows the results averaged over the different motion directions in the first interval. In the stimulus 
A
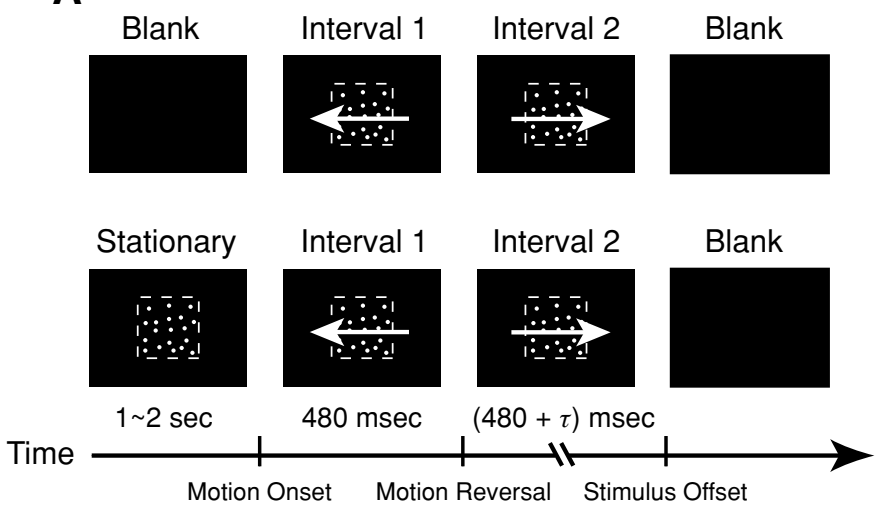

B

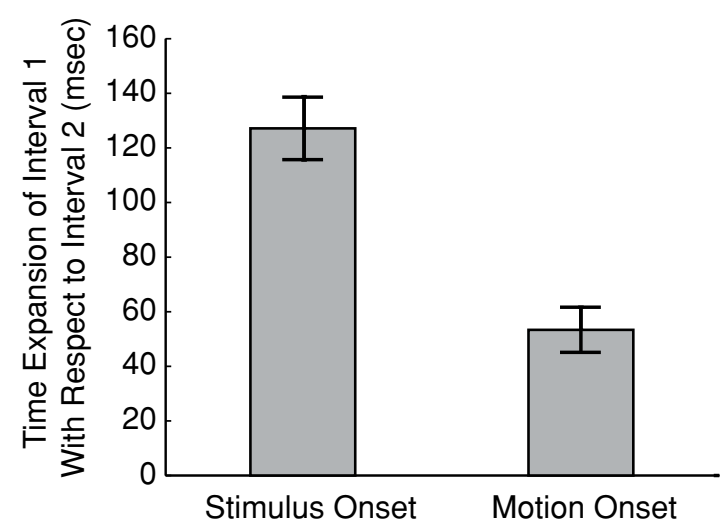

Figure 1. The basic phenomenology of the onset time expansion illusion. (A) The typical stimuli are illustrated. In the stimulus onset condition (upper panels), a random dot motion stimulus appeared abruptly from a blank display. The onset of the motion was synchronous with the stimulus onset, and it moved for $480 \mathrm{msec}$ (Interval 1). Then, the stimulus reversed direction and continued to move for a variable duration, $(480+\tau)$ msec (Interval 2). The motion direction was thus presented either in the leftward-rightward or rightward-leftward order. In the motion onset condition (lower panels), a stationary stimulus was presented before the motion began. Otherwise the stimulus was identical to that used in the stimulus onset condition. In both cases, Interval 1 was perceived to be longer in time than Interval 2. (B) The magnitude of the time expansion of Interval 1 with respect to Interval 2 for the stimulus onset condition (left) and the motion onset condition (right). The data were obtained using a method employing constant stimuli in which the duration of Interval 2 was varied, whereas the duration of Interval 1 was constant. The data are the means from four observers. Error bars indicate $\pm 1 S E M$.

onset and motion onset conditions, the first interval was perceived to be longer than the second interval. However, the effect was much larger in the stimulus onset condition, in which the first interval was preceded by a blank display $[F(1,3)=383.4, p<.001]$. In this condition, the second interval needed to be physically extended by $127.2 \mathrm{msec}$ ( $\pm 11.6 \mathrm{msec} S E M$ ) to match the duration of the first interval in percept. On the other hand, in the motion onset condition, when the same stimulus was preceded by a stationary stimulus ( $>1 \mathrm{sec})$, the effect was smaller ( $53.3 \mathrm{msec} \pm$ $8.3 \mathrm{msec} S E M)$, but still significantly larger than zero $[t(3)=6.42, p<.01]$. This difference in the magnitude suggests that the type of stimuli marking the onset of an interval plays a critical role in time perception.

\section{EXPERIMENT 2 Multiple-Interval Experiment}

The finding that the first interval was perceived to be longer than the second could be explained both by the expansion of the first interval and by the shrinkage of the second interval. The finding that the effect size depends on the onset condition (i.e., blank or stationary) suggests that a change in the perceived duration of the first interval 
rather than the second was responsible for the time distortion. However, the possibility of the time of the second interval shrinking (or the last vanishing stimulus) cannot be ruled out. Thus, in the following experiment, we addressed the question of to what degree the effect was expansion of the first interval, or shrinking of the last interval.

To do so, we used a variable number of intervals, rather than just two, as in the previous experiments. The number of intervals was varied between two, three, four, and five. The observers were asked to compare the duration of the last interval and the interval just before the last, and to indicate which interval was perceptually longer.

\section{Method}

Subjects. Four subjects participated. One of the subjects was an author (R.K.), but the other 3 observers were naive as to the purpose of the experiments. All of the observers had normal or corrected-tonormal visual acuity.

Procedure. The number of intervals was varied between two, three, four, and five. When the number of intervals was two, the condition was identical to the corresponding condition in the previous experiment. When there were more intervals, the stimulus moved horizontally back and forth. The duration of all the intervals except the last one was fixed at $480 \mathrm{msec}$, and the duration of the last interval was varied between $-213,-107,-53,-26,0,26,53,107$, and $213 \mathrm{msec}$, with respect to the preceding interval $(480 \mathrm{msec})$. The task was to compare the duration of the last interval with the preceding interval and to indicate which interval was longer in time. Twenty samples were obtained per condition. For example, when the number of intervals was four, the duration of the fourth (i.e., the last) interval was varied, and the observers had to compare the duration of the fourth interval with that of the third. Only the stimulus onset condition was tested, and thus there were a total of four conditions, with nine timings each. Each condition was separated into two blocks. The order of the total of eight blocks was randomized across observers.

Design. The PSEs for each of the four conditions were compared in a one-factor repeated measures design.

\section{Results and Discussion}

The results are shown in Figure 2. Changing the number of intervals had a significant effect on the amount of time expansion $[F(3,9)=102.75, p<.001]$. The time expansion was observed only in the two-interval condition, in which the first interval was compared with the second interval. When a second or later interval was compared with the succeeding interval, no time expansion was found. Instead, those intermediate intervals were consistently judged as slightly shorter than the last interval. In other words, there was a slight tendency for the final interval to expand, with respect to the previous one.

Our initial motivation for this experiment was to see whether the shift in the PSE is due to time expansion of the first interval or time shrinking of the second interval. With regard to this question, our results unequivocally show that the bulk of the effect is due to an overestimation of the duration of the first interval. Thus, we refer to this effect as the time expansion illusion, or TEI, hereafter.

A similar pattern of time distortion in the visual domain has been reported in other types of stimuli (Rose \& Summers, 1995). In their study, Rose and Summers found
A
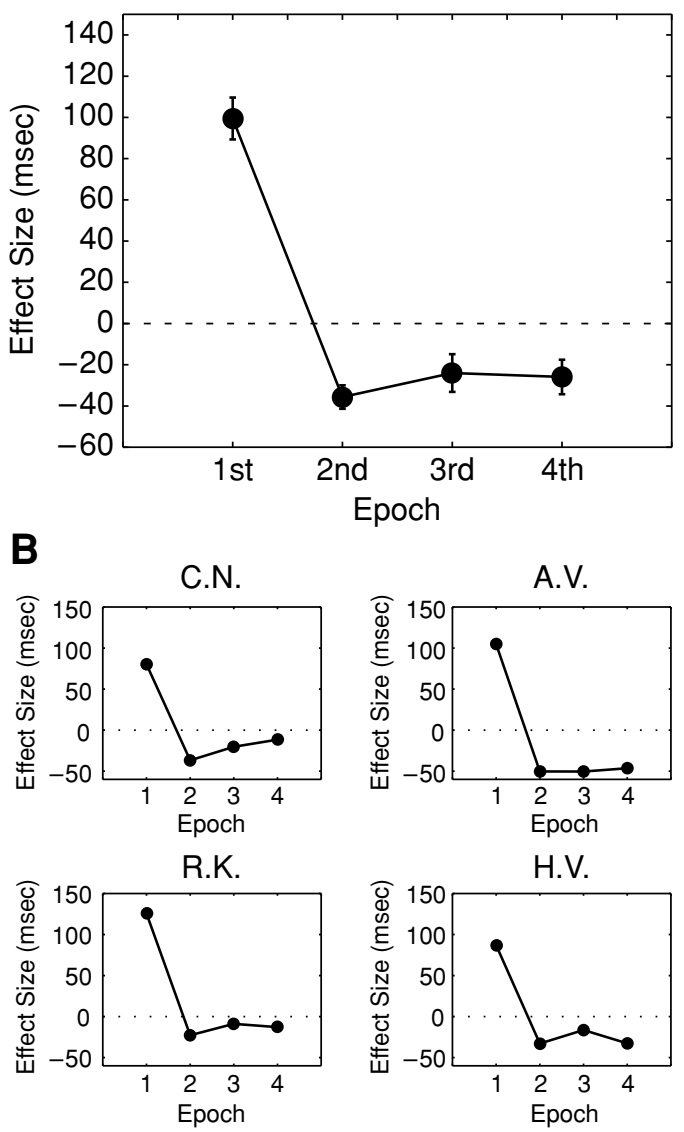

Figure 2. The results of the multiple-interval experiment. (A) The mean perceived duration of the preceding interval with respect to the last interval $(N=4)$. Error bars indicate $\pm 1 S E M$. (B) The results of the individual observers.

that when a train of four visual flashes of equal duration $(\sim 600 \mathrm{msec})$ is presented, the duration of the first flash is perceived to be longer. In order for the first flash to be perceived as being of equal duration, it has to be physically shortened by about $200 \mathrm{msec}$, on average. They also found that the last, fourth flash is perceived to be slightly longer in comparison with the third. This pattern of time expansion - a large expansion for the first interval and a minor expansion for the last interval-is consistent with the results of our experiment. Thus, it is tempting to infer that similar mechanisms are involved both in the time expansion found by Rose and Summers and the time expansion reported in the present study.

\section{EXPERIMENT 3 Standard-Duration Experiment}

As we stated earlier, the origin of perceptual time distortion can be categorized into two classes under the framework of SET. First, a time distortion can be caused by different pulse rates for the first and second intervals. 
For example, if the internal clock were to tick faster for the first interval than for the second interval, the first interval would be judged to be longer than the second. If this were the underlying mechanism for the TEI, the effect size should scale, perhaps proportionally, with the standard duration. Second, the TEI could be due to a systematic shift of the temporal markers opening the switch in response to a stimulus onset or motion onset. The time markers for the two intervals consist of stimulus onset (or motion onset), motion reversal, and stimulus offset. A constant difference in the latency for those time markers may be responsible for the TEI. If this were the case, the effect size would be constant regardless of the standard duration.

To examine these possibilities, we tested whether the magnitude of the TEI is proportional to the standard duration (480 $\mathrm{msec}$, in the previous experiment), or a constant increment of perceived duration, regardless of the standard stimulus duration.

\section{Method}

Subjects. Four subjects participated. One of the subjects was an author (R.K.), but the other 3 observers were naive as to the purpose of the experiments. All observers had normal or corrected-to-normal visual acuity.

Procedure. Three durations of the first interval were used (240, 480 , and $960 \mathrm{msec}$ ). The duration of the second interval was varied between $-213,-107,-53,-26,0,26,53,107$, and $213 \mathrm{msec}$, with respect to the first interval. The task was to indicate which interval was longer in time. The direction of motion in the first interval was randomized between leftward or rightward. A total of 40 samples were made for each duration of the second interval.

Both the stimulus onset and motion onset conditions were tested. As in Experiment 1, both the duration of the blank and the duration of the stationary stimulus were randomly chosen between 1 and $2 \mathrm{sec}$ (using uniform sampling).

Design. The subjects were tested individually in each condition, thus producing a $2 \times 3$ within-subjects factorial design, with onset type (stimulus onset or motion onset) and duration of the first interval $(240,480$, and $960 \mathrm{msec})$ as factors. Three base durations and two initial conditions (i.e., stimulus onset and motion onset) resulted in a total of six conditions. These different conditions were tested in separate blocks, and the order was randomized across observers. The experiment took about $3 \mathrm{~h}$ per observer, including the breaks between blocks.

\section{Results and Discussion}

The results are shown in Figure 3. The effect size of the TEI was more or less constant across the different durations of the first interval. Repeated measures ANOVA showed a main effect of onset condition $[F(1,6)=55.3$, $p<.01$ ], whereas the duration of the first interval was inconsequential $[F(2,6)=2.10, p=.20]$. Also, no significant interaction was found between the two factors $[F(2,6)<1]$. This indicates that the time expansion is a constant addition of perceived duration to, rather than a proportional scaling of, the duration of the first interval.

The main effect of the onset condition is consistent with our Experiment 1. When the stimulus appears abruptly, the first interval is perceived to be longer than when a stationary stimulus preceded. More pertinent to the original aim of this experiment is the lack of differences between

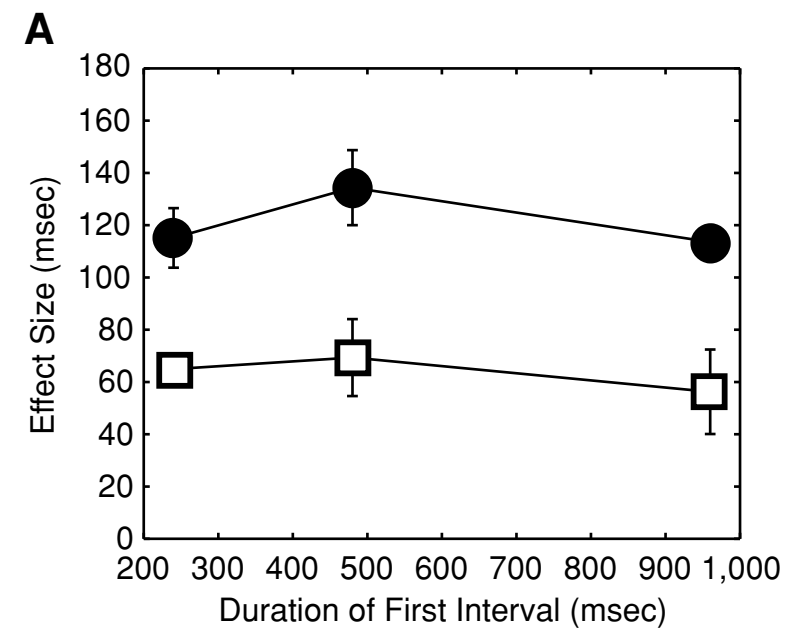

B

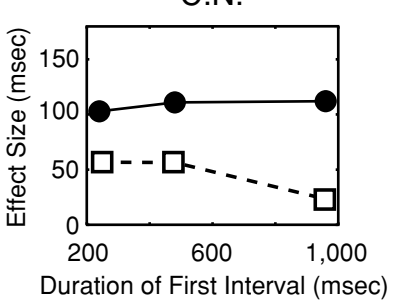

R.K.
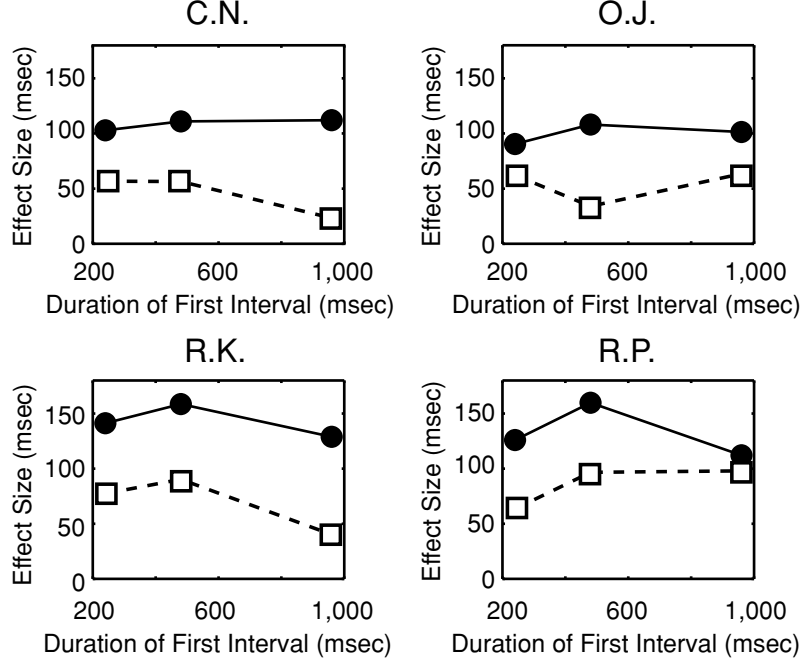

Figure 3. The results of the motion duration experiment. (A) The mean effect size $(N=4)$ is shown as a function of the duration of Interval 1. The solid circles indicate the results of the stimulus onset condition, and the open squares the results of the motion onset condition. Error bars indicate $\pm 1 S E M$. (B) The results of the individual observers.

different standard durations. This indicates that the time expansion results from the addition of a set amount of time to the first interval, as opposed to a scaled expansion of the whole first interval. Thus, our results support the idea that the different onset conditions affect the timing of opening the gate from the pulse generator to the accumulator in the SET model. If the different pulse rates were the cause of the TEI, the magnitude would scale (at least partially) in proportion to the standard duration.

\section{EXPERIMENT 4A RTs to Stimulus Onset and Motion Onset}

On the basis of the results in Experiment 3, it appears that the TEI can be accounted for by different processing 
delays for setting time markers (opening the gate) at the beginning of a time interval. However, other explanations are possible. For example, a higher level of attention elicited in one condition might cause the pulse rate to increase for only a brief moment. In particular, if the increased pulse rate lasted less than $240 \mathrm{msec}$ (the shortest standard interval tested in Experiment 3), the constant increase in the TEI magnitude could be fully explained.

To further examine the account based on differential processing delays between stimulus onset and motion onset, we tried to measure processing latency more directly. To this end, we measured the simple reaction times (RTs) to stimulus onset and motion onset. Moreover, we compared the simple RTs with the choice RTs in a direction discrimination task using the same set of stimuli. If one of the two experiments revealed a difference that matched the difference in duration estimates in the experiments so far, this comparison would be informative with regard to the level of visual processing at which the switch is opened.

\section{Method \\ Subjects \\ Four subjects participated. One of the subjects was an author (R.K.), but the other 3 observers were naive as to the purpose of the experiments. All of the observers had normal or corrected-to-normal visual acuity.}

\section{Procedure}

Simple reaction task. We used the first intervals of Experiment 1 as stimuli for this experiment. As in the basic experiment, there were two conditions: the stimulus onset and motion onset conditions. In the stimulus onset condition, a moving stimulus appeared suddenly. The observers were asked to respond with a keypress as soon as they saw the stimulus. In the motion onset condition, a stationary stimulus appeared at the beginning of each trial. After a random duration $(1-2 \mathrm{sec})$ of viewing the stationary stimulus, the stimulus started moving. The observers were asked to respond to the motion onset as quickly as possible. In both conditions, the direction of the motion was randomized. Three separate blocks were performed for each condition. Each block consisted of 40 trials. Thus, each observer had a total of 120 trials per condition.

Motion discrimination task. In a separate experiment, the same observers participated in another RT experiment in which they were again required to respond to the onset for the stimulus onset and motion onset conditions. However, in this experiment, they were required to judge the direction of the motion (left or right), and had to press a corresponding key as quickly as possible.

\section{Design}

The simple reaction task and the discrimination task were treated as separate experiments. For each experiment, the mean RTs for each onset condition were compared in a within-subjects design using a paired $t$ test.

\section{Results and Discussion}

The results for the simple reaction task are shown in Figure 4. Simple RT was significantly faster for the stimulus onset than for the motion onset $[t(3)=11.07, p<$ $.001]$. The difference in RT ranged around 40-60 msec, which roughly corresponds to the difference in the time expansion between the stimulus onset and motion onset conditions, supporting the latency hypothesis.

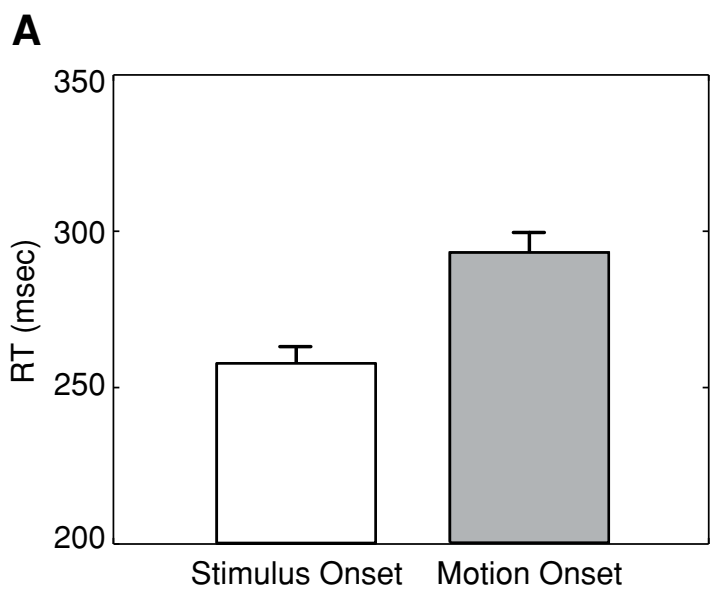

B
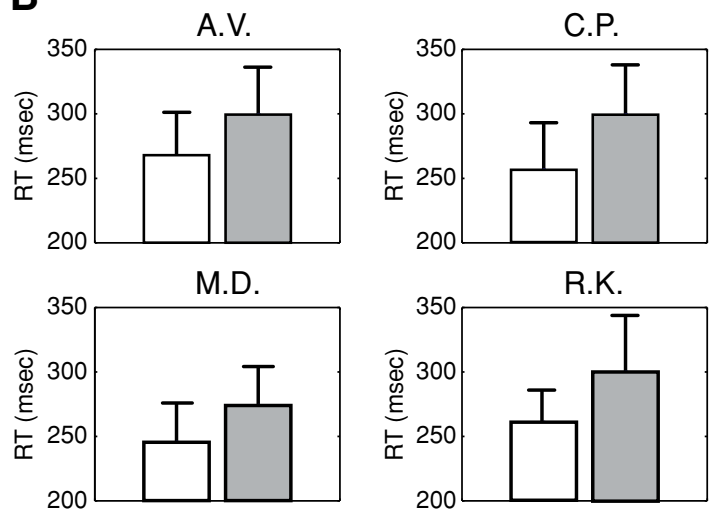

Figure 4. The results of the simple RT task (Experiment 4A). (A) The mean RT of 4 observers is shown for each onset condition. The white bar shows the results of the stimulus onset condition, and the gray bar shows the results of the motion onset condition. Error bars indicate $1 S E M$. (B) The results of the individual observers. Error bars indicate 1 standard deviation $(S D)$.

On the other hand, we did not find a difference in the RT for the motion discrimination task (Figure 5). No significant difference was found for any of the individual subjects $[t(3)=0.0334, p>.05]$. This indicates that the processing latency necessary for judging the motion direction does not differ between the two onset types.

These findings suggest that the opening of the switch occurs at the moment when the initial, perhaps the earliest, response to a stimulus is produced, rather than when all the features of a stimulus are fully processed.

\section{EXPERIMENT 4B RTs to Motion Reversal and Stimulus Offset}

Although the results of Experiment 4A support the idea that the difference in the TEI size between the stimulus onset and the motion onset conditions is due to differential processing latency, it is unclear whether the idea of processing latency can fully explain why the first interval is perceived to be longer than the second. In our stimuli, the first interval is the interval between stimulus (or motion) onset 
A

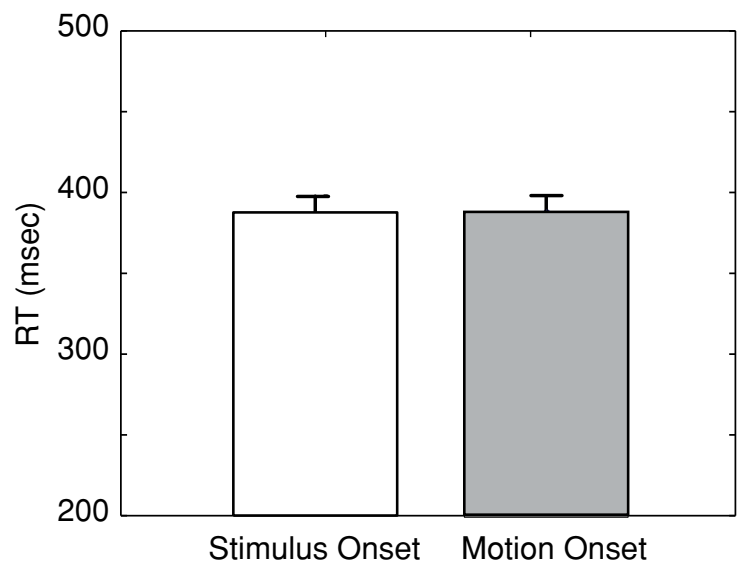

B

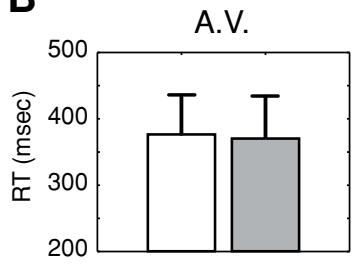

M.D.
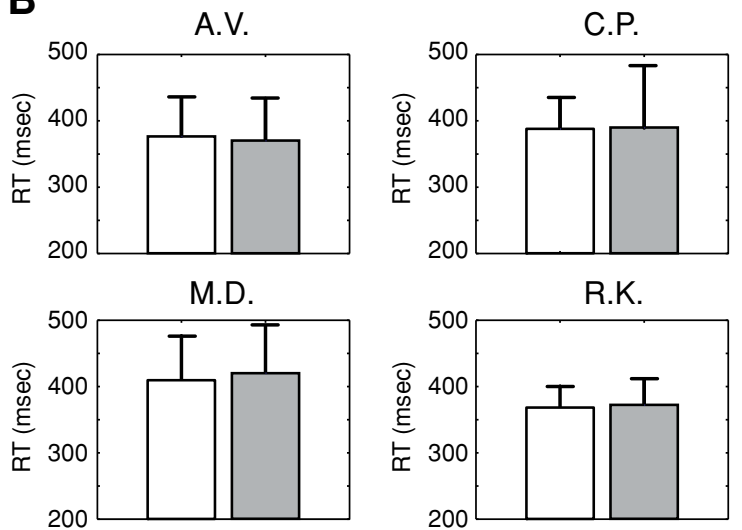

Figure 5. The results of the direction discrimination task (Experiment 4A). (A) The mean RT of 4 observers is shown for each onset condition. The white bar shows the results of the stimulus onset condition, and the gray bar shows the results of the motion onset condition. Error bars indicate $1 S E M$. (B) The results of the individual observers. Error bars indicate $1 S D$.

and motion reversal, and the second interval is between the motion reversal and stimulus offset. In other words, there are three temporal events defining the two intervals: stimulus (or motion) onset, motion reversal, and stimulus offset. The latency hypothesis predicts the following relationship:

$$
\left(T_{\text {rev }}-T_{\text {on }}\right)=\left(T_{\text {off }}-T_{\text {rev }}\right)+\tau,
$$

where the left-hand side denotes the perceived duration of the first interval and the right-hand side the perceived duration of the second interval. $\tau$ is the amount of TEI — that is, the duration of the first interval relative to the second interval. $T_{\text {on }}$ denotes the processing latency for the onset, $T_{\text {rev }}$ the latency for the reversal, and $T_{\text {off }}$ the latency for the offset.

In this experiment, we investigated whether this relation holds when the processing latencies for motion reversal and stimulus offset are estimated by simple RTs.

\section{Method}

Subjects. Four subjects participated. One of the subjects was an author (R.K.). The other 3 subjects were naive as to the purpose of the experiments, and they did not participate in Experiment 4A. All of the observers had normal or corrected-to-normal visual acuity.

Procedure. We measured the RT for stimulus onset, motion onset, motion reversal, and stimulus offset. The task and stimuli for the first two tasks (i.e., stimulus onset and motion onset) were identical to the simple reaction tasks in Experiment 4A. For the motion reversal, a moving stimulus appeared, and after the subjects had viewed the moving stimulus for a random duration $(1-2 \mathrm{sec})$, the stimulus reversed its direction and moved for another $480 \mathrm{msec}$. The observers were asked to respond to the motion reversal as quickly as possible with a keypress. For the motion offset condition, the moving stimulus disappeared after a random viewing duration (1-2 sec) from its onset. In all four conditions, the direction of the motion was randomized.

Design. The RTs for each of the four conditions were calculated for individual subjects. Thus, a one-factor within-subjects design was employed. In one block, only one of the four conditions was tested. Each block consisted of 40 trials, and each subject performed two blocks per condition. The order of the eight blocks (4 types of events $\times 2$ blocks) was randomized for each subject.

\section{Results and Discussion}

The results for the simple reaction task are shown in Figure 6 . The RTs differed significantly depending on the type of event the observers had to respond to $[F(3,9)=$ $37.72, p<.001]$. The mean RTs were 255.2, 292.2, 313.7, and $280.7 \mathrm{msec}$ for the stimulus onset, motion onset, motion reversal, and stimulus offset conditions, respectively.

On the basis of these data, we constructed diagrams to account for the TEI (Figure 7). Figure 7A depicts the stimulus onset condition. Assuming that there is no change in the internal clock speed, and that the processing time associated with motor commands is constant for all the RT tasks, we can compute the perceived duration for the intervals as follows. Here, we denote the constant duration of the first and second interval as $T$. The first interval is calculated as the processed timing of motion reversal minus the processed time of stimulus onset. This is $(T+314) \mathrm{msec}-(255) \mathrm{msec}$, i.e., $(T+59) \mathrm{msec}$. Similarly, the perceived duration of the second interval is calculated as the timing of motion offset $(2 T+281) \mathrm{msec}$ minus the time of motion reversal $(T+314)$ msec. This is $(T-33)$ msec. By comparing these two estimates of perceived durations, we obtain $(T+59)-(T-33)=$ $92 \mathrm{msec}$, which predicts that the first interval should be perceived as being $92 \mathrm{msec}$ longer than the second interval. Similarly, the first interval defined by a motion onset is $55 \mathrm{msec}$ longer than the second interval (Figure 7B). These estimates are in reasonable agreement with the amount of time expansion obtained in the previous experiments (122 $\mathrm{msec}$ for the stimulus onset condition and $63 \mathrm{msec}$ for motion onset; the mean of all data in Experiment 1 and Experiment 3).

However, this analysis cannot accommodate the results of Experiment 2 - that is, the time expansion of the last interval when there are more than two intervals. On the contrary, the processing latency hypothesis predicts that the final interval should become $33 \mathrm{msec}$ shorter than the preceding interval. One possible explanation for this discrepancy is that the processing latency for the motion reversal is reduced when there are multiple reversals. In 

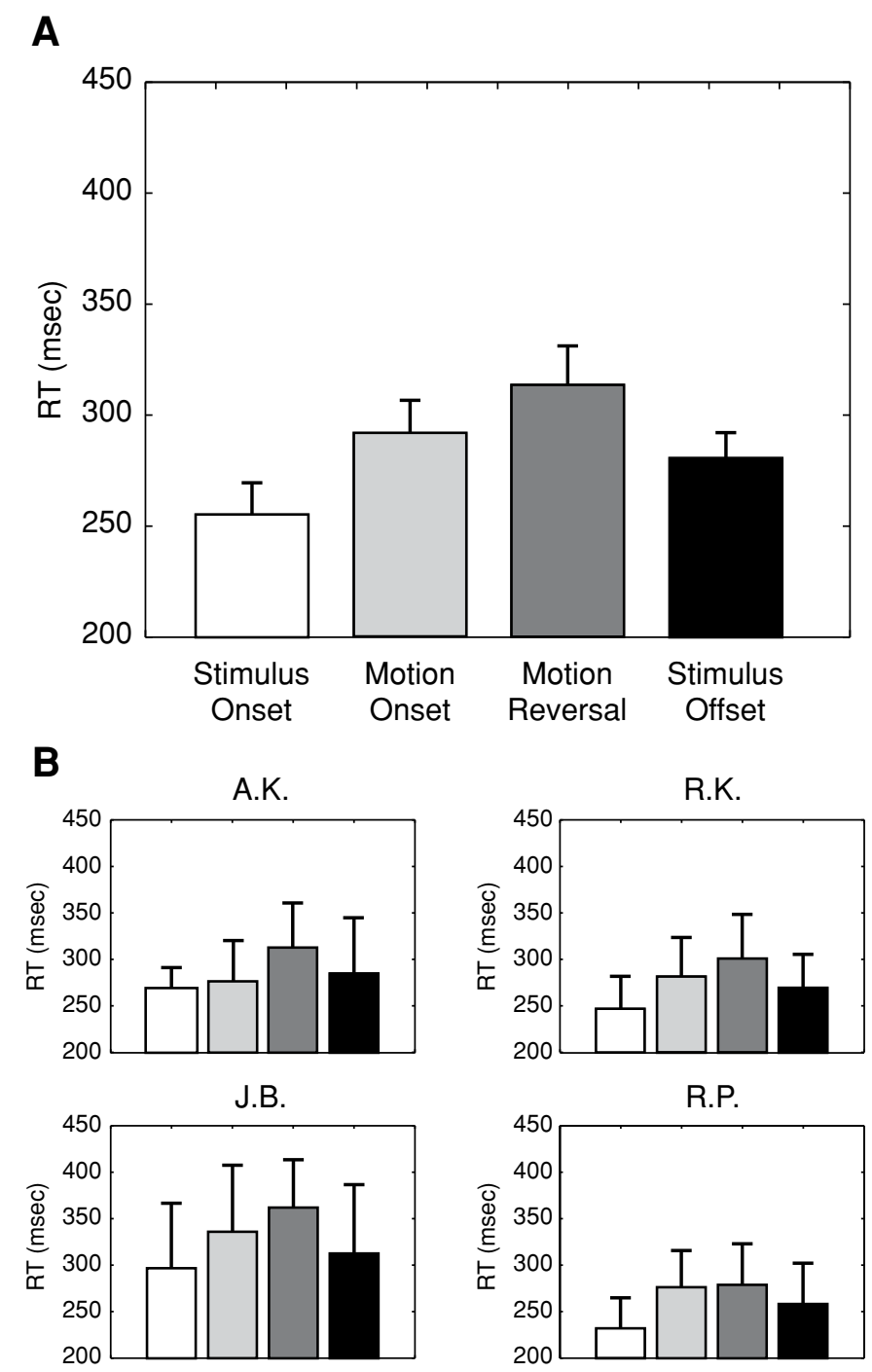

Figure 6. The results of the simple RT experiment (Experiment 4B). (A) The mean RT of 4 observers is shown for each condition. The white bar shows the results for the stimulus onset condition, the gray bar shows the results for the motion onset condition, the darker gray bar shows the results for the motion reversal condition, and the black bar shows the results for the motion offset condition. Error bars indicate 1 SEM. (B) The results of the individual observers. Error bars indicate $1 S D$.

particular, the reversals occurred every $480 \mathrm{msec}$ in Experiment 2 . This sort of rhythmicity may have provided a predictive temporal cue, facilitating the processing of motion reversals (Jones \& Boltz, 1989).

\section{GENERAL DISCUSSION}

In the present study, we demonstrated a novel time distortion illusion. When a stimulus appears abruptly, its duration is perceived as being longer relative to when it appears following a stationary array. The magnitude of the TEI is a roughly constant addition of time, independent of the base interval durations. It was about $120 \mathrm{msec}$ for the stimulus onset condition, and $60 \mathrm{msec}$ for the motion onset condition. Thus, the difference in the TEI magnitude for the two types of onset conditions was about $60 \mathrm{msec}$.

\section{Time-Order Error}

Duration judgment between two time intervals embedded in a sequence of intervals is generally systematically biased. The biasing influence that presentation order has on the perceived duration of multiple stimuli is known as time-order error (TOE; see Hellström, 1985). The TOE can be either positive or negative, depending on the durations and methods. In the stimulus durations used in our study, the duration of a first interval is typically judged as being longer than a second interval of the same duration, because negative TOE is typically found for durations of 


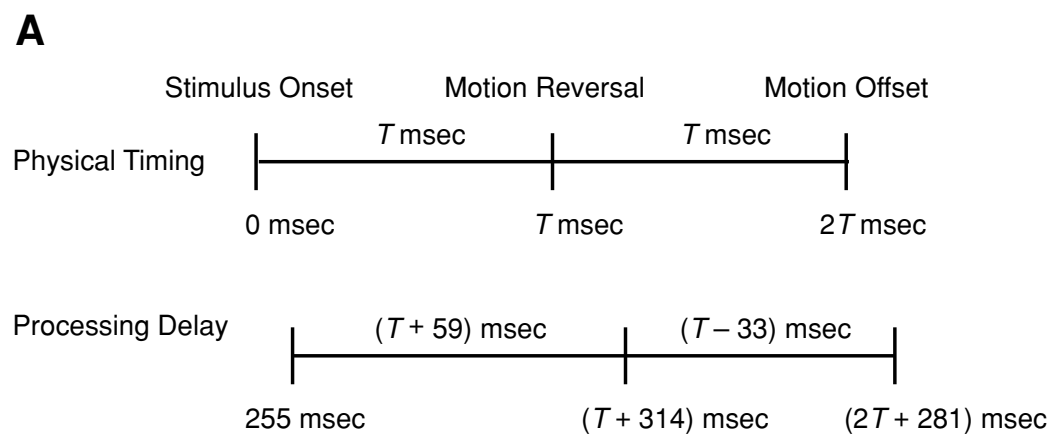

First interval is 92 msec longer.

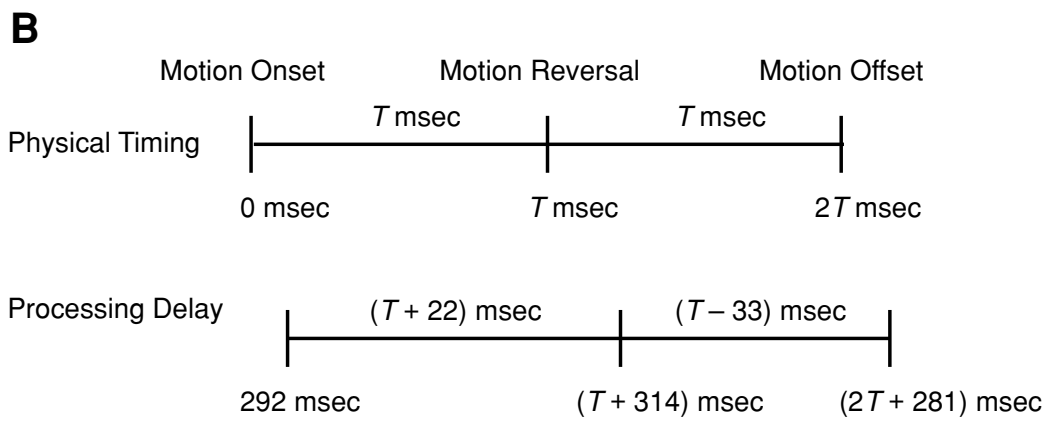

First interval is $55 \mathrm{msec}$ longer.

Figure 7. Schematic illustrations of the processing latency hypothesis. (A) The TEI in the stimulus onset condition. (B) The TEI in the motion onset condition.

seconds (Allan, 1977, 1979; Allan \& Gibbon, 1994; Jamieson \& Petrusic, 1975; Walker \& Scott, 1981; Wearden $\&$ Ferrara, 1993). This systematic bias is consistent with our finding; in the TEI, the perceived duration of the first interval is longer than that of the second interval.

Generally, the TOE decreases when the base duration increases (Allan, 1977). However, such a systematic change was not found when the base duration was varied (Experiment 3). Moreover, the TEI size depended on the type of onset. Time expansion was much larger when the first interval began with an abrupt stimulus onset than when it was preceded by a stationary stimulus. Although a form of TOE could contribute a fixed amount to both conditions, the presentation order per se cannot explain the difference between the onset conditions. Thus, the stimulus onset seems to play a critical role in the time expansion of the first interval.

\section{Chronostasis}

Our findings are also reminiscent of another time illusion, called chronostasis, whereby the first interval (1 sec) after a saccadic eye movement is perceived to be longer than the subsequent events with the same physical duration (Yarrow et al., 2001). Chronostasis is induced not only by saccades, but also by other types of voluntary actions, such as pressing a button (Park, Schlag-Rey, \& Schlag, 2003). Because, in our experiments, trials were initiated by observers' voluntary action (a keypress), it may appear that the time expansion we report here can be subsumed as just a variation of chronostasis. Moreover, the effect size of chronostasis is about the same magnitude as is the TEI. In the effective conditions, the first intervals become about $120 \mathrm{msec}$ longer in both cases.

Given the similarities between the TEI and chronostasis, the commonalities and differences in the induction conditions need to be closely examined. In all of the experiments conducted on chronostasis so far (Alexander et al., 2005; Hodinott-Hill, Thilo, Cowey, \& Walsh, 2002; Jackson et al., 2005; Park et al., 2003; Yarrow et al., 2001; Yarrow, Haggard, \& Rothwell, 2004; Yarrow, Johnson, Haggard, \& Rothwell, 2004; Yarrow \& Rothwell, 2003), the first interval - which is subject to time expansion-was always preceded by another stimulus (e.g., a digit 0 ). Such a configuration corresponds to our motion onset conditions. However, these were the conditions in which we did not find a large time expansion.

As to the time expansion in chronostasis that is related to action, the effect has been found to disappear when the first event after an action is delayed by $500 \mathrm{msec}$. In our experiments, the observers' trigger for a trial and stimulus onset were separated by at least $1 \mathrm{sec}$; this temporal separation should have effectively removed the chronostasis component of the time expansion. Indeed, the small amount of time expansion observed in our motion onset 
condition quantitatively matches the results of control conditions in control data for chronostasis experiments in which action (e.g., eye movement) was not involved (Yarrow et al., 2001). Given that sufficient temporal separation between action and stimulus onset eliminates the chronostasis components, the TEI seems to have a different origin than chronostasis. Whereas chronostasis is induced by voluntary actions, the TEI is driven by the stimulus configuration. The difference in their induction conditions further supports the hypothesis of different origins for these two types of time expansion.

\section{Models of Time Perception}

We will discuss the results under the framework offered by the SET of time perception. We consider two possibilities in which a perceived duration could be distorted: first, differential latencies for opening and closing the gate controlled by a switch, and second, a change in the rate of the pulses generated by a clock.

Before we discuss the results in terms of the first type of time distortion, which we favor, we examine to what degree our results might be accounted for by changes in the pulse rate. One of the possible sources for a change in the pulse rate is a different attentional level for different onset types. Generally, attention is known to increase the perceived duration of events. This is often accounted for as follows: When more attention is poured into the time estimation, more temporal cues accumulate, and therefore the perceived duration becomes longer. This effect of attention is supported by interference experiments. In a dual-task condition, in which observers were engaged in another concurrent task, the same duration was estimated to be shorter than in a single-task condition, in which attention was fully directed to a time estimation task (Brown, 1985, 1995, 1997; Brown \& Stubbs, 1992; Coull et al., 2004; DeWolfe \& Duncan, 1959; Hicks, Miller, Gaes, \& Bierman, 1977). Also, when an oddball stimulus is embedded in a sequence of more common stimuli, the perceived duration of the oddball is expanded (Tse et al., 2004). This effect, termed time's subjective expansion, is also accounted for by the enhanced attention to the oddball stimulus.

Is the TEI reported here just another instance of time expansion by enhanced attention? This appears to be likely, given the fact that abrupt stimulus onset (Posner, 1980; Yantis \& Jonides, 1984) and motion onset (Franconeri \& Simons, 2003; see also Hillstrom \& Yantis, 1994) attract attention. The onset of the first interval would attract attention in comparison with the second, and this may have resulted in a time expansion of the first interval relative to the second.

Although this idea seems broadly consistent with our results, our experiments showed the limitations of the attentional account. In order to make this model compatible with our results, the attentional effect would need to operate entirely within a brief time window. If the attentional effect persists longer than $240 \mathrm{msec}$ after the beginning of the interval (the smallest standard duration tested in Experiment 3), the TEI would have increased with longer standard durations. However, our Experiment 3 showed that this is not the case.

We therefore attribute the TEI to a timing difference for opening the gate, because of the hypothesis' simplicity and quantitative consistency with our RT results. Our RT data for the simple reaction task suggest that the processing time for the onset of a stimulus occurs earlier than the processing time for the onset of motion. This implies that there is a differential processing latency for the initial onset between the two conditions. In fact, the differences in the RT (40 60 msec) approximately match the differences in the time expansion (50 70 $\mathrm{msec})$. Moreover, experiments on the visual evoked potential (VEP) provide a further insight on this issue. For stimulus onset and motion onset, VEP takes a distinct time course (Torriente, Valdes-Sosa, Ramirez, \& Bobes, 1999). The first VEP responses to stimulus onset and motion onset are N125 and N170, respectively. The latency differences for these responses are approximately $45 \mathrm{msec}$, which accords with the RT data (37 msec; Experiment 4) as well as our time estimation data (59 $\mathrm{msec}$; mean of all data in Experiment 1 and Experiment 3; see Figure 1B and Figure 3). Taken together, the results of the RT and VEP experiments show that the processing latency for stimulus onset and motion onset is consistent with the difference in the TEI magnitude induced by the respective onset types. Manipulation of stimulus contrast and speed is known to result in a systematic change in the RT for motion onset (Burr, Fiorentini, \& Morrone, 1998; López-Moliner, 2005). This provides an opportunity to further test the latency hypothesis by examining whether subjective time estimation follows the systematic changes in the RT.

\section{REFERENCES}

Alexander, I., Thilo, K. V., Cowey, A., \& Walsh, V. (2005). Chronostasis without voluntary action. Experimental Brain Research, 161, 125-132.

Allan, L. G. (1977). The time-order error in judgments of duration. Canadian Journal of Psychology, 31, 24-31.

Allan, L. G. (1979). The perception of time. Perception \& Psychophysics, 26, 340-354.

Allan, L. G., \& Gibbon, J. (1994). A new temporal illusion or the TOE once again? Perception \& Psychophysics, 55, 227-229.

Arao, H., Suetomi, D., \& Nakajima, Y. (2000). Does time-shrinking take place in visual temporal patterns? Perception, 29, 819-830.

BLOCK, R. A. (1982). Temporal judgments and contextual change. Journal of Experimental Psychology: Learning, Memory, \& Cognition, 8, 530-544.

Brainard, D. H. (1997). The Psychophysics Toolbox. Spatial Vision, 10, 433-436.

Brown, S. W. (1985). Time perception and attention: The effects of prospective versus retrospective paradigms and task demands on perceived duration. Perception \& Psychophysics, 38, 115-124.

Brown, S. W. (1995). Time, change, and motion: The effects of stimulus movement on temporal perception. Perception \& Psychophysics, 57, 105-116.

Brown, S. W. (1997). Attentional resources in timing: Interference effects in concurrent temporal and nontemporal working memory tasks. Perception \& Psychophysics, 59, 1118-1140.

Brown, S. W., \& StubBs, D. A. (1992). Attention and interference in prospective and retrospective timing. Perception, 21, 545-557.

Burr, D. C., Fiorentini, A., \& Morrone, C. (1998). Reaction time to motion onset of luminance and chromatic gratings is determined by perceived speed. Vision Research, 38, 3681-3690. 
Coull, J. T., Vidal, F., Nazarian, B., \& Macar, F. (2004). Functional anatomy of the attentional modulation of time estimation. Science, 303, 1506-1508.

Creelman, C. D. (1962). Human discrimination of auditory duration. Journal of the Acoustical Society of America, 34, 582-593.

DeWolfe, R. K. S., \& Duncan, C. P. (1959). Time estimation as a function of level of behavior of successive tasks. Journal of Experimental Psychology, 58, 153-158.

Franconeri, S. L., \& Simons, D. J. (2003). Moving and looming stimuli capture attention. Perception \& Psychophysics, 65, 999-1010.

GibBon, J. (1977). Scalar expectancy theory and Weber's law in animal timing. Psychological Review, 84, 279-325.

Gibbon, J., Church, R. M., \& Meck, W. H. (1984). Scalar timing in memory. In J. Gibbon and L. Allan (Eds.), Timing and time perception (Annals of the New York Academy of Sciences, Vol. 423, pp. 52-77). New York: New York Academy of Sciences.

Gibson, J. J. (1975). Events are perceivable but time is not. In J. T. Fraser $\&$ N. Lawrence (Eds.), The study of time II: Proceedings of the second conference of the International Society for the Study of Time (pp. 295301). New York: Springer.

Grondin, S., Ivry, R. B., Franz, E., Perreault, L., \& Metthé, L. (1996). Markers' influence on the duration discrimination of intermodal intervals. Perception \& Psychophysics, 58, 424-433.

Grondin, S., \& Rousseau, R. (1991). Judging the relative duration of multimodal short empty time intervals. Perception \& Psychophysics, 49, 245-256.

Hellström, А. (1985). The time-order error and its relatives: Mirrors of cognitive processes in comparing. Psychological Bulletin, 97, 35-61.

Hicks, R. E., Miller, G. W., Gaes, G., \& Bierman, K. (1977). Concurrent processing demands and the experience of time-in-passing. American Journal of Psychology, 90, 431-446.

Hillstrom, A. P., \& Yantis, S. (1994). Visual motion and attentional capture. Perception \& Psychophysics, 55, 399-411.

Hodinott-Hill, I., Thilo, K. V., Cowey, A., \& Walsh, V. (2002). Auditory chronostasis: Hanging on the telephone. Current Biology, 12, 1779-1781.

Jackson, S. R., Newport, R., Osborne, F., Wakely, R., Smith, D., \& WALSH, V. (2005). Saccade-contingent spatial and temporal errors are absent for saccadic head movements. Cortex, 41, 205-212.

Jamieson, D. G., \& Petrusic, W. M. (1975). Pairing effects and timeorder errors in duration discrimination. Perception \& Psychophysics, 18, 107-113.

Jones, M. R., \& Boltz, M. (1989). Dynamic attending and responses to time. Psychological Review, 96, 459-491.

Kanai, R., Paffen, C. L. E., Hogendoorn, H., \& Verstraten, F. A. J. (2006). Time dilation in dynamic visual display. Journal of Vision, $\mathbf{6}$, 1421-1430.

KanaI, R., Sheth, B. R., \& Shimojo, S. (2004). Stopping the motion and sleuthing the flash-lag effect: Spatial uncertainty is the key to perceptual mislocalization. Vision Research, 44, 2605-2619.

LóPEZ-Moliner, J. (2005). Reaction time asymmetries between expansion and contraction. Psicológica, 26, 139-146.

Nakajima, Y., ten Hoopen, G., Hilkhuysen, G., \& Sasaki, T. (1992). Time-shrinking: A discontinuity in the perception of auditory temporal patterns. Perception \& Psychophysics, 51, 504-507.

Nakajima, Y., ten Hoopen, G., Sasaki, T., Yamamoto, K., Kadota, M., Simons, M., \& Suetomi, D. (2004). Time-shrinking: The process of unilateral temporal assimilation. Perception, 33, 1061-1079.
NaKajima, Y., ten Hoopen, G., \& VAn der Wilk, R. (1991). A new illusion of time perception. Music Perception, 8, 431-448.

PARK, J., Schlag-Rey, M., \& Schlag, J. (2003). Voluntary action expands perceived duration of its sensory consequence. Experimental Brain Research, 149, 527-529.

Pelli, D. G. (1997). The VideoToolbox software for visual psychophysics: Transforming numbers into movies. Spatial Vision, 10, 437-442.

Posner, M. I. (1980). Orienting of attention. Quarterly Journal of Experimental Psychology, 32, 3-25.

Poynter, W. D. (1989). Judging the duration of time intervals: A process of remembering segments of experience. In I. Levin \& D. Zakay (Eds.), Time and human cognition: A life-span perspective (pp. 305331). Amsterdam: Elsevier.

RAmmsayer, T. H., \& Leutner, D. (1996). Temporal discrimination as a function of marker duration. Perception \& Psychophysics, 58, 1213-1223.

RaYmond, J. E. (2003). New objects, not new features, trigger the attentional blink. Psychological Science, 14, 54-59.

Rose, D., \& Summers, J. (1995). Duration illusions in a train of visual stimuli. Perception, 24, 1177-1187.

Torriente, I., Valdes-Sosa, M., Ramirez, D., \& Bobes, M. A. (1999). Visual evoked potentials related to motion-onset are modulated by attention. Vision Research, 39, 4122-4139.

Treisman, M. (1963). Temporal discrimination and the indifference interval: Implications for a model of the "internal clock." Psychological Monographs: General \& Applied, 77(13, Whole No. 576), 1-31.

Tse, P. U., Intriligator, J., Rivest, J., \& Cavanagh, P. (2004). Attention and the subjective expansion of time. Perception \& Psychophysics, 66, 1171-1189.

WALKER, J. T., \& SCOTT, K. J. (1981). Auditory-visual conflicts in the perceived duration of lights, tones, and gaps. Journal of Experimental Psychology: Human Perception \& Performance, 7, 1327-1339.

Wearden, J. H., \& Ferrara, A. (1993). Subjective shortening in humans' memory for stimulus duration. Quarterly Journal of Experimental Psychology, 46B, 163-186.

YANTIS, S., \& JonidES, J. (1984). Abrupt visual onsets and selective attention: Evidence from visual search. Journal of Experimental Psychology: Human Perception \& Performance, 10, 601-621.

Yarrow, K., Haggard, P., Heal, R., Brown, P., \& Rothwell, J. C. (2001). Illusory perceptions of space and time preserve cross-saccadic perceptual continuity. Nature, 414, 302-305.

Yarrow, K., Haggard, P., \& Rothwell, J. C. (2004). Action, arousal, and subjective time. Consciousness \& Cognition, 13, 373-390.

Yarrow, K., Johnson, H., Haggard, P., \& Rothwell, J. C. (2004). Consistent chronostasis effects across saccade categories imply a subcortical efferent trigger. Journal of Cognitive Neuroscience, 16, 839-847.

YArrow, K., \& Rothwell, J. C. (2003). Manual chronostasis: Tactile perception precedes physical contact. Current Biology, 13, 11341139.

ZAKAY, D. (1993). Relative and absolute duration judgments under prospective and retrospective paradigms. Perception \& Psychophysics, 54, 656-664.

(Manuscript received January 27, 2005; revision accepted for publication November 1, 2005.) 